\title{
The centrosome: a multifaceted cellular weapon against chromosome instability
}

\author{
Giulia Guarguaglini • Daniela Cimini
}

Published online: 23 December 2015

(C) Springer Science+Business Media Dordrecht 2015

\begin{abstract}
A Special Issue on Centrosomes and Chromosome Stability cannot start in any other way but by mentioning the "father" of the field, Theodor Boveri, who anticipated over a century ago many of the issues we are discussing today (Ried 2009; Scheer 2014). He described and named the centrosome, proposed it as the central organelle of cell division and chromosome segregation (Boveri 1887, 1901), and also proposed that supernumerary centrosomes may be a cause of malignancy (Boveri 1914, 2008). It was not until decades after Boveri's observations and theories that the scientific community re-discovered the centrosome. This renewed interest has led to a wealth of studies on the regulation of centrosome structure and function and its contribution to tumorigenesis (Bornens and Gonczy 2014; Gonczy 2015).

The most obvious and recognized centrosome function is microtubule nucleation and organization. Based on this widely acknowledged function, several studies in
\end{abstract}

Responsible Editor: Beth A. Sullivan.

G. Guarguaglini $(\bowtie)$

Institute of Molecular Biology and Pathology, CNR National Research Council, c/o Department of Biology and Biotechnology, Sapienza University of Rome, Via degli Apuli 4, 00185 Rome, Italy

e-mail: giulia.guarguaglini@uniroma1.it

D. Cimini $(\square)$

Department of Biological Sciences and Biocomplexity Institute, Virginia Tech, 1015 Life Science Circle, Blacksburg, VA 24061, USA

e-mail: cimini@vt.edu the 1990s focused on the role of centrosomes in spindle assembly and chromosome segregation or on the role of supernumerary centrosomes in driving aneuploidy and cancer (Salisbury et al. 1999; Winey 1999; Zimmerman et al. 1999). Unexpected observations then led into an era of skepticism about the centrality of centrosomes in mitosis (Doxsey 2001; Hyman 2000; Raff 2001). Indeed, centrosome requirement for mitotic spindle assembly was challenged by the findings that (i) centrosome ablation in vertebrate cells does not impair bipolar spindle formation and mitotic progression (Hinchcliffe et al. 2001; Khodjakov et al. 2000), (ii) spindles assemble around chromatin-loaded beads in Xenopus egg extracts in the absence of centrosomes (Heald et al. 1996), and (iii) most strikingly, flies with strongly impaired centrosome function (Megraw et al. 2001) or devoid of centrioles (Basto et al. 2006) develop to adulthood without major problems. Moreover, it was argued that multipolar divisions may not produce viable progeny, challenging the view of extra-centrosomes as drivers of tumorigenesis through multipolar spindle assembly (Nigg 2002), but leaving the unanswered question as to what enables cancer cells to tolerate or possibly even select for the presence of extra centrosomes.

Together, these puzzling observations and new ideas fueled the exploration of additional centrosomal functions, both in interphase and mitosis (Rieder et al. 2001), and the investigation of the routes to stably amplified centrosomes in cancer (Godinho et al. 2009), thus leading to novel important discoveries. Among non-mitotic centrosomal functions, impressive advances were made in the field of cilia formation and function in non- 
dividing cells, leading to key discoveries on the molecular bases of ciliopathies (Chavali et al. 2014; Nigg and Raff 2009). In mitosis, centrosomes were shown to be key players in determining spindle orientation and controlling symmetric/asymmetric cell division (Giansanti et al. 2001; Khodjakov and Rieder 2001; Tang and Marshall 2012). A major finding elucidating the connection between centrosome abnormalities and cancer was the centrosome clustering mechanism, through which supernumerary centrosomes coalesce into two spindle poles prior to anaphase onset, thus suppressing multipolar division in cells carrying extra-centrosomes (Basto et al. 2008; Kwon et al. 2008; Quintyne et al. 2005). Importantly, two subsequent studies evidenced that the transient multipolar state that precedes centrosome clustering causes an increase in merotelic kinetochore attachments and hence anaphase lagging chromosomes (Ganem et al. 2009; Silkworth et al. 2009), thus providing the missing link between extra-centrosomes and the chromosomal instability typically observed in cancer cells. Moreover, a number of recent studies showed that even in the presence of a correct centrosome number, temporal mis-regulation of certain centrosomal processes (e.g., delayed or accelerated centrosome separation upon mitotic entry) can also affect the accuracy of chromosome segregation (Kaseda et al. 2012; McHedlishvili et al. 2012; Nam and van Deursen 2014; Silkworth et al. 2012). Overall, data reported so far indicate that, although dispensable for cell division under many circumstances, when present, centrosomes behave dominantly with respect to other microtubule organizing pathways. Thus, even though spindle assembly can be achieved in the absence of centrosomes due to redundant mechanisms, the dominant behavior of centrosomes explains the strong impact of centrosome aberrations on the fidelity of chromosome segregation.

Given these recent advances and the emerging evidence that centrosomes play an important role in keeping chromosome stability in check, we decided that the time was right for a Special Issue on this topic. Overall, this Special Issue focuses on the links between various aspects of centrosome function and chromosome stability. The issue includes nine review articles, organized in three broad groups.

The first three contributions provide an integrated view of the different processes controlled by centrosomes - throughout the cell cycle - that are relevant to genome stability, and of the mechanisms that act to ensure coordination of centrosome- and chromosome- dependent processes. Dorothy Lerit and John Poulton open the issue by providing an extensive overview of the cellular processes that more strictly rely on the presence of centrosomes, focusing on those that may impact genome stability, and discussing how this varies in different organisms or tissues. They highlight how centrosomal processes and cell cycle progression are strongly interconnected and signal to each other to ensure the proper unfolding of events eventually leading to balanced chromosome segregation at cell division. The contribution of centrosomes to the final stage of the cell cycle as spindle pole organizers is then developed by Patrick Meraldi. After revisiting the studies that challenged the importance of centrosomes for spindle assembly, he summarizes recent evidence supporting the view that, although not strictly required, centrosomes provide a kinetic advantage to the mitotic process and protect cells from chromosomal instability. Meraldi further discusses the key role of centrosomes in controlling other aspects of spindle assembly and function, particularly focusing on spindle positioning both in asymmetric and symmetric cell division. An additional way-independent of spindle organization and function - in which centrosomes contribute to genome stability is through their intriguing link to the DNA damage response, which is discussed in a contribution by Lisa Mullee and Ciaran Morrison. Although the connection between centrosomes and the DNA damage response, along with the view of the centrosome as a signaling center, was proposed over a decade ago (Lange 2002; Sibon et al. 2000), the underlying mechanisms are still being actively investigated. A coverage of this topic seems particularly timely, as further understanding of the mechanisms linking the centrosome to DNA damage response pathways will not only broaden our knowledge of cell cycle regulation, but also provide critical insight for therapeutic anti-cancer applications, where DNA damaging agents are commonly used.

The sophisticated temporal co-regulation of centrosomal and cell cycle processes strongly relies on signaling pathways; this is the focus of the second group of articles in this Special Issue. The contribution by Patrizia Lavia discusses emerging evidence that the RAN GTPase acts as a master regulator of centrosome function, ensuring spatial coordination of virtually all centrosomal processes, particularly through the antagonistic relation with its major effector importin beta on downstream cargos. Protein kinases are also widely used to control cell cycle processes and are involved in 
regulation of centrosome structure and function. A contribution by Jan van Deursen and collaborators describes the impressive advances made in the past few years in elucidating the phosphorylation cascades that regulate the two steps of centrosome separation (i.e., disengagement and movement), how such regulation ensures chromosome stability, and the perspective application of this information for cancer research and therapy. Finally, Ying Zhang and Paul Galardy explore the accumulating evidence of a local action of the ubiquitin/proteasome machinery at centrosomes and the importance of protein degradation for the regulation of certain centrosome processes, such as duplication and separation.

The last three articles are intended to illustrate the current view on the link between centrosome aberrations and disease. Amanda Bolgioni and Neil Ganem focus on the long-standing issue of supernumerary centrosomes in cancer and describe the emerging evidence that inactivation of the Hippo tumor suppressor pathway may represent an adaptive mechanism that allows cancer cells to tolerate extra-centrosomes. Next, Marco Raffaele Cosenza and Alwin Krämer lead us into the mechanistic aspects of supernumerary centrosomes and chromosomal instability in cancer and the potential therapeutic approaches based on modulation of such phenotypes. Although centrosome aberrations are historically associated with cancer, their link to developmental diseases has also become widely acknowledged. The closing article of this Special Issue, by Maddalena Nano and Renata Basto, discusses the apparent paradox of centrosomal defects contributing to over-proliferation in cancer and reduced proliferation in development, and presents this problem from a tissue- and organismspecific perspective.

We would like to conclude by thanking all the contributors for enthusiastically accepting the invitation to be part of this project and for making the assembly of this Special Issue such an interesting and enriching experience. We would also like to express our appreciation to the executive editor Dr. Beth Sullivan for initially reaching out to us with the idea and for masterfully coordinating the production process.

\section{References}

Basto R, Lau J, Vinogradova T, Gardiol A, Woods CG, Khodjakov A, Raff JW (2006) Flies without centrioles. Cell 125: 1375-1386
Basto R, Brunk K, Vinadogrova T, Peel N, Franz A, Khodjakov A, Raff JW (2008) Centrosome amplification can initiate tumorigenesis in flies. Cell 133:1032-1042

Bornens M, \& Gonczy P (2014) Centrosomes back in the limelight. Philos Trans R Soc Lond B Biol Sci 369

Boveri $\mathrm{T}$ (1887) Ueber den antheil des spermatozoon an der teilung des eies. Sitzungsber Ges Morph Physiol Müchen 3: 151-164

Boveri T (1901) Zellenstudien IV. Über die natur der centrosomen. Jena Zeitschr Naturw 35:1-220

Boveri T (1914) Zur Frage der Entstehung maligner Tumoren. Jena, Gustav Fischer Verlag

Boveri T (2008) Concerning the origin of malignant tumours by Theodor Boveri. Translated and annotated by Henry Harris. J Cell Sci 121(Supplement 1):1-84

Chavali PL, Putz M \& Gergely F (2014) Small organelle, big responsibility: the role of centrosomes in development and disease. Philos Trans R Soc Lond B Biol Sci 369

Doxsey S (2001) Re-evaluating centrosome function. Nat Rev Mol Cell Biol 2:688-698

Ganem NJ, Godinho SA, Pellman D (2009) A mechanism linking extra centrosomes to chromosomal instability. Nature 460 : 278-282

Giansanti MG, Gatti M, Bonaccorsi S (2001) The role of centrosomes and astral microtubules during asymmetric division of Drosophila neuroblasts. Development 128:1137-1145

Godinho SA, Kwon M, Pellman D (2009) Centrosomes and cancer: how cancer cells divide with too many centrosomes. Cancer Metastasis Rev 28:85-98

Gonczy P (2015) Centrosomes and cancer: revisiting a longstanding relationship. Nat Rev Cancer 15:639-652

Heald R, Tournebize R, Blank T, Sandaltzopoulos R, Becker P, Hyman A, Karsenti E (1996) Self-organization of microtubules into bipolar spindles around artificial chromosomes in Xenopus egg extracts. Nature 382:420-425

Hinchcliffe EH, Miller FJ, Cham M, Khodjakov A, Sluder G (2001) Requirement of a centrosomal activity for cell cycle progression through G1 into S phase. Science 291: $1547-1550$

Hyman AA (2000) Centrosomes: Sic transit gloria centri. Curr Biol 10:R276-R278

Kaseda K, Mcainsh AD, Cross RA (2012) Dual pathway spindle assembly increases both the speed and the fidelity of mitosis. Biol Open 1:12-18

Khodjakov A, Rieder CL (2001) Centrosomes enhance the fidelity of cytokinesis in vertebrates and are required for cell cycle progression. J Cell Biol 153:237-242

Khodjakov A, Cole RW, Oakley BR, Rieder CL (2000) Centrosome-independent mitotic spindle formation in vertebrates. Curr Biol 10:59-67

Kwon M, Godinho SA, Chandhok NS, Ganem NJ, Azioune A, Thery M, Pellman D (2008) Mechanisms to suppress multipolar divisions in cancer cells with extra centrosomes. Genes Dev 22:2189-2203

Lange BM (2002) Integration of the centrosome in cell cycle control, stress response and signal transduction pathways. Curr Opin Cell Biol 14:35-43

Mchedlishvili N, Wieser S, Holtackers R, Mouysset J, Belwal M, Amaro AC, Meraldi P (2012) Kinetochores accelerate centrosome separation to ensure faithful chromosome segregation. J Cell Sci 125:906-918 
Megraw TL, Kao LR, Kaufman TC (2001) Zygotic development without functional mitotic centrosomes. Curr Biol 11: $116-120$

Nam HJ, van Deursen JM (2014) Cyclin B2 and p53 control proper timing of centrosome separation. Nat Cell Biol 16: $538-549$

Nigg EA (2002) Centrosome aberrations: cause or consequence of cancer progression? Nat Rev Cancer 2:815-825

Nigg EA, Raff JW (2009) Centrioles, centrosomes, and cilia in health and disease. Cell 139:663-678

Quintyne NJ, Reing JE, Hoffelder DR, Gollin SM, Saunders WS (2005) Spindle multipolarity is prevented by centrosomal clustering. Science 307:127-129

Raff JW (2001) Centrosomes: central no more? Curr Biol 11: R159-R161

Ried T (2009) Homage to Theodor Boveri (1862-1915): Boveri's theory of cancer as a disease of the chromosomes, and the landscape of genomic imbalances in human carcinomas. Environ Mol Mutagen 50:593-601

Rieder CL, Faruki S, Khodjakov A (2001) The centrosome in vertebrates: more than a microtubule-organizing center. Trends Cell Biol 11:413-419
Salisbury JL, Whitehead CM, Lingle WL, Barrett SL (1999) Centrosomes and cancer. Biol Cell 91:451-460

Scheer U (2014) Historical roots of centrosome research: discovery of Boveri's microscope slides in Wurzburg. Philos Trans R Soc Lond B Biol Sci 369

Sibon OC, Kelkar A, Lemstra W, Theurkauf WE (2000) DNAreplication/DNA-damage-dependent centrosome inactivation in Drosophila embryos. Nat Cell Biol 2:90-95

Silkworth WT, Nardi IK, Scholl LM, Cimini D (2009) Multipolar spindle pole coalescence is a major source of kinetochore mis-attachment and chromosome mis-segregation in cancer cells. PLoS One 4:e6564

Silkworth WT, Nardi IK, Paul R, Mogilner A, Cimini D (2012) Timing of centrosome separation is important for accurate chromosome segregation. Mol Biol Cell 23:401-411

Tang N, Marshall WF (2012) Centrosome positioning in vertebrate development. J Cell Sci 125:4951-4961

Winey M (1999) Cell cycle: driving the centrosome cycle. Curr Biol 9:R449-R452

Zimmerman W, Sparks CA, Doxsey SJ (1999) Amorphous no longer: the centrosome comes into focus. Curr Opin Cell Biol 11:122-128 